\title{
The Synaptic Organization of the Claustral Projection to the Cat's Visual Cortex
}

\author{
Nuno Maçarico da Costa, David Fürsinger, and Kevan A. C. Martin \\ Institute for Neuroinformatics, University of Zürich and Eidgenössische Technische Hochschule Zürich, 8057 Zürich, Switzerland
}

The claustrum is a subcortical structure reciprocally connected with most areas of neocortex. This strategic location suggests an integrative role of the claustrum across different sensory modalities. However, our knowledge of the synaptic relationship between the neocortex and the claustrum is basic. In this study, we address this question through a structural investigation of the claustral projection to the ipsilateral primary visual cortex of the cat. Light microscopic reconstructions of axons from the entire thickness of cortex showed a very sparse innervation of the entire cortical depth, with most synaptic boutons in layers $2 / 3$ and 6 . Axons bearing numerous boutons terminaux and boutons en passant branched in these laminae. The sparse innervation did not seem to be compensated by particularly large synapses, given that the postsynaptic densities in the superficial layers are of comparable sizes $\left(0.1 \mu \mathrm{m}^{2}\right)$ to other cortical synapses. All claustral synapses were asymmetric and in most cases targeted spines ( $87 \%$ in layer $4,94 \%$ in layers $2 / 3$ and $97 \%$ in layer 6 ). The pattern of innervation together with the known physiology of this projection suggests that the claustrum has a modulatory effect on visual cortex.

\section{Introduction}

The claustrum is thought to be connected to most cortical areas, an idea that was born from tracing experiments using degeneration techniques, in cats, rabbits, and monkeys (Carman et al., 1964; Narkiewicz, 1964; Kemp and Powell, 1970). These results were then reproduced in many other mammal species, including the tractography evidence from humans (Morys et al., 1993; FernándezMiranda et al., 2008). Injections in the claustrum also labeled numerous subcortical structures such as the putamen, zona incerta, dorsomedial thalamus, the suprageniculate thalamus, and hypothalamus (LeVay and Sherk, 1981b; Markowitsch et al., 1984; Erickson and Lewis, 2004; Yoshida et al., 2006) (but see Mathur et al., 2009). This comprehensive connectivity is well suited to its name, which is derived from monastic architecture and refers to the broad passage that gives access to all the buildings and apartments of a monastery. Not surprisingly, the claustrum has been suggested to be involved in cross-modal processing (Ettlinger and Wilson, 1990) and as a place where the different sensory modalities are integrated and bound, playing an important role in conscious perception, cognition, and action (Crick and Koch, 2005). The pattern and targets of the synaptic connections of the claustrum to visual cortex are currently unresolved and is the subject of this study.

In the cat, the visual claustrum is located at the dorsocaudal end of the nucleus and contains a single, orderly map of the contralateral visual hemifield (Olson and Graybiel, 1980; LeVay and Sherk, 1981a). At least five visual areas project to the claustrum $(17,18,19,21 \mathrm{a}$, and PMLS) and are expected to contribute

Received June 17, 2010; revised Aug. 2, 2010; accepted Aug. 7, 2010.

This work was supported by European Union Daisy Project Grant FP6-2005-015803. We thank Rita Bopp for her expert technical assistance and John Anderson for his helpful comments on the manuscript.

Correspondence should be addressed to Kevan A. C. Martin, Institute for Neuroinformatics, University of Zürich and ETH Zürich, Wintherthurerstrasse 190, 8057 Zürich, Switzerland. E-mail: kevan@ini.phys.ethz.ch.

DOI:10.1523/JNEUROSCI.3122-10.2010

Copyright $\odot 2010$ the authors $\quad 0270-6474 / 10 / 3013166-05 \$ 15.00 / 0$ to this map (LeVay and Sherk, 1981b). According to radioactive tracer studies, claustral afferents target all laminae of area 17, but seem to be more prominent in layers 4 and 6 (LeVay and Sherk, 1981b; LeVay, 1986). This is a feature that is typical of "feedforward" projections (LeVay and Gilbert, 1976; Leventhal, 1979; Kawano, 1998; Anderson et al., 2009) and is consistent with the hypothesis that the claustrum drives its cortical targets (Crick and Koch, 2005). Claustral axons form type 1 synapses (Gray, 1959) in the visual cortex, mostly with spines in layers 1 and 6 , while in layer 4 dendritic shafts and spines are equal synaptic targets (LeVay, 1986). The projection to layer $2 / 3$ has not been examined and the projection to layer 5 has been reported to be barely visible.

Crick and Koch (2005) suggested that to understand the function of the claustrum one should study its structure. Here, we follow their advice and examine at the ultrastructural level, the claustral cortical pathway. We labeled the dorsocaudal visual claustrum by discrete injections of biotinylated dextran amine (BDA) and analyzed its projection to area 17 at both light and electron microscopic levels. Reconstructions, innervation patterns and quantification of targets were used to complement the analysis.

\section{Materials and Methods}

The material presented here was taken from two cats (one male and one female). The experimental protocols for the project "Microcircuits of Neocortex" were approved by the Canton of Zurich Veterinary Office, which issued the license No. 50/3002 to K.A.C.M. Details of the anesthetics and surgical operation have been reported previously (da Costa and Martin, 2009b).

The claustrum is a very slender nucleus and, therefore, it is easily missed stereotaxically, even though its position is well described (Clarke and Henderson, 1914). Thus, we located the claustrum electrophysiologically using the strategy devised by LeVay and Sherk (1981b). We first determined the anterior border of the dorsal lateral geniculate nucleus (dLGN) by recording with a varnished tungsten electrode. This corresponds to the anteroposterior limit of the caudal end of the claustrum. By moving stepwise anteriorly and laterally, we located the visual 
A
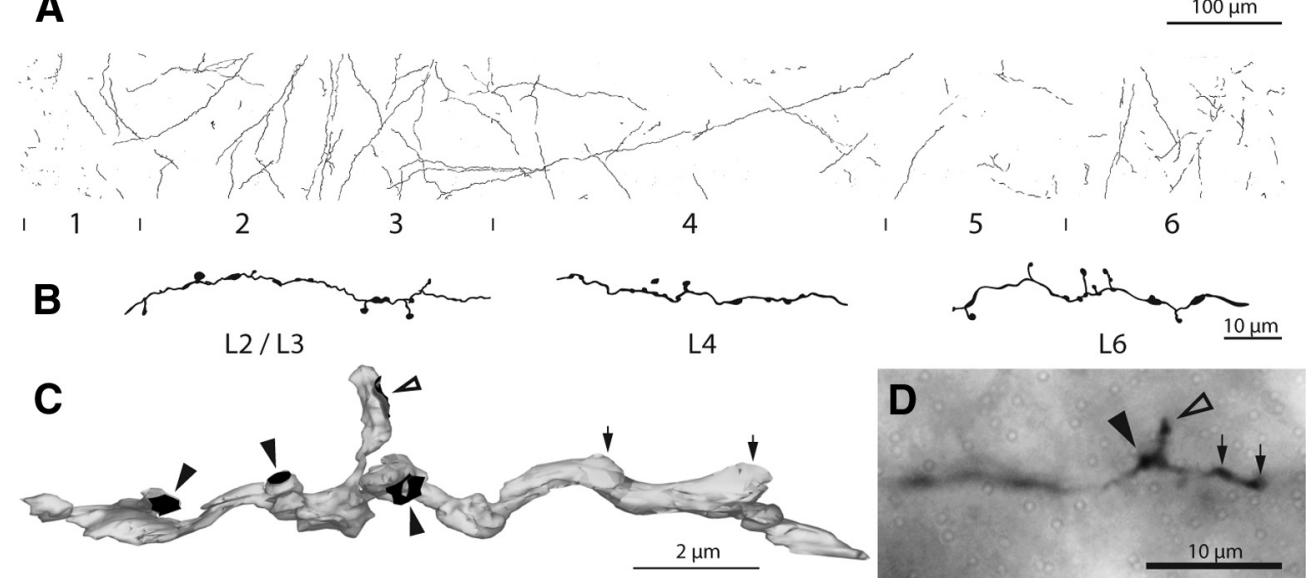
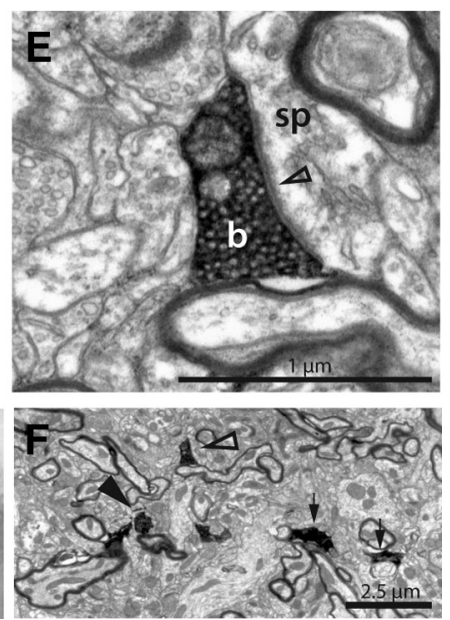

Figure 1. Innervation of area 17 by claustral axons. $\boldsymbol{A}$, Light microscopic reconstruction of all the axons innervating a strip of area 17. Laminae and their boundaries are indicated below. $\boldsymbol{B}$, High-power drawing of three sections of labeled claustral axon taken from different layers. C, Three-dimensional reconstruction from serial ultrathin sections of a labeled claustral axon (gray) in layer 4 showing 3 synapses formed by boutons en passant (solid arrowheads) and 1 synapse from a bouton terminaux (open arrowhead). The two swellings (small arrows) do not contain vesicles and are filled with mitochondria (see also supplemental Fig. 2, available at www.jneurosci.org as supplemental material). $\boldsymbol{D}$, Light micrograph of corresponding axon (in $\boldsymbol{C}$ ) showing the bouton terminaux (open arrowhead), one bouton en passant (solid arrowhead), and the two swellings (small arrows) that could be misleadingly interpreted as synaptic boutons by only considering light micrographs. $\boldsymbol{E}$, High-power electron micrograph of the vesicle filled bouton terminaux (b) of $\boldsymbol{C}$ and $\boldsymbol{D}$ forming an asymmetric synapse (open arrowhead) with a spine (sp). $\boldsymbol{F}$, Low-power electron micrograph of identified axon collateral shown in $\mathbf{C}$ and $\boldsymbol{D}$; arrows as in $\boldsymbol{D}$.

segment of the claustrum by visual responses recorded at a depth of $\sim 10$ $\mathrm{mm}$ below the surface of the brain. Additionally, a visual field map of the claustrum (LeVay and Sherk, 1981a) was used to determine and correct the recording position. Once the claustrum was found, the tungsten electrode was replaced by a biotinylated dextran amine (BDA) (MW10,000, Invitrogen)-filled glass micropipette and the recordings were repeated to ensure the pipette tip (diameter, $10-18 \mu \mathrm{m}$ ) was located in the correct position in the claustrum. Ionophoretic injections were made for 5 min at 2-4 $\mu \mathrm{A}$. The BDA was delivered as a $10 \%$ solution in $0.05 \mathrm{M}$ Tris plus $0.2 \mathrm{M} \mathrm{KCl}$. Three injections were made in one animal and eight injections in the second. The injections were made in close proximity. After a 7-9 d survival period, the animals underwent an acute recording procedure under general anesthesia, before being very deeply anesthetized with an intravenous injection of Saffan $(20 \mathrm{mg} / \mathrm{kg})$ and perfused transcardially with a normal saline solution, followed by a solution of $4 \%$ paraformaldehyde, $0.3 \%$ glutaraldehyde, and $15 \%$ picric acid in $0.1 \mathrm{M} \mathrm{PB} \mathrm{pH} \mathrm{7.4.} \mathrm{The} \mathrm{brain} \mathrm{was} \mathrm{blocked} \mathrm{in} \mathrm{a} \mathrm{transverse} \mathrm{stereotaxic}$ plane and the block was sunk in a series of sucrose solutions of 10,20, and $30 \%$ in $0.1 \mathrm{M} \mathrm{PB}$ to serve as cryoprotectant. The blocks were then freezethawed in liquid nitrogen and washed in $0.1 \mathrm{M} \mathrm{PB}$. Sections were cut from the blocks at $80 \mu \mathrm{m}$ in the coronal plane and collected in $0.1 \mathrm{M} \mathrm{PB}$. After washes in TBS, the sections were left overnight $\left(5^{\circ} \mathrm{C}\right)$ in an avidin-biotin complex (ABC kit Elite, Vector Laboratories). The peroxidase activity was identified using 3,3-diaminobenzidene tetrahydrochloride (SigmaAldrich) with nickel intensification. After assessment by light microscopy (LM), selected regions of tissue were treated with $1 \%$ osmium tetroxide in $0.1 \mathrm{M} \mathrm{PB}$. Dehydration through alcohols ( $1 \%$ uranyl acetate in the $70 \%$ alcohol) and propylene oxide allowed flat mounting in Durcupan (Fluka Chemie) on glass slides.

Sections were first analyzed using light microscopy. Reconstructions of segments of the axon were drawn manually using a drawing tube attachment to the light microscope. Reconstructions were made using a $100 \times$ objective magnification under oil. Serial ultrathin sections from regions of interest were then collected at $60 \mathrm{~nm}$ thickness on Pioloformcoated, single-slot copper grids. Synapse classification in the EM followed conventional criteria (Gray, 1959). Type 1 synapses were classified as asymmetric when a postsynaptic density and round vesicles were identified and type 2 synapses as symmetric when a presynaptic membrane mirrored a postsynaptic specialization and when the bouton contained flattened vesicles. Electron micrographs of synapses were taken at $25,000 \times$. Three-dimensional reconstructions were made with Reconstruct (Fiala, 2005).

\section{Results}

\section{Light microscopy}

Analysis of the histological sections showed that all injection sites were in the visual claustrum (supplemental Fig. 1, available at www.jneurosci.org as supplemental material). In ipsilateral area 17 , labeled fibers ascended radially from the white matter to the surface of the cortex. Many axons appeared to be parallel to the coronal plane of section. The axons were difficult to trace as they were very thin (range of axon diameters found in ultrathin sections, $0.06-0.5 \mu \mathrm{m})$ and in parts lightly stained. The axons formed both boutons terminaux and boutons en passant (Fig. 1; supplemental Fig. 1, available at www.jneurosci.org as supplemental material). Very few branches were observed on the path of the axons. To estimate which were the principal cortical layers innervated by claustral axons, vertical strips of cortex were reconstructed (Fig. $1 A$; supplemental Fig. $1 E$, available at www.jneurosci. org as supplemental material). In these reconstructions, all axons were drawn within a strip of cortex, $80 \mu \mathrm{m}$ thick, that extended from the white matter to the surface of the brain, passing through all layers.

Cortical layer $2 / 3$ hosted the majority of axons and boutons. In one, animal layer 6 was equally well innervated. Layer 1 was also innervated in both animals. Layers 4 and 5 contained few labeled axons although the ascending axons passing through layers 4 and 5 always formed a few boutons en passant.

The frequency of occurrence of stained axons was quite low. The dLGN innervation of primary visual cortex forms $\sim 6 \%$ of the excitatory synapses in layer 4 (da Costa and Martin, 2009a). The claustral innervation of layer 4 looks insignificant when compared with the already low percentage of the dLGN innervation. The sparseness of boutons impeded random statistical analysis such as counting synapses at random locations.

Light microscopic reconstructions of single axons as well as reconstructions of vertical strips of innervated cortex feature a change in bouton arrangements. The boutons change quite abruptly from both en passant and terminaux in the deep layers to only en passant boutons in layer 4 (Fig. 1). The same change in bouton morphology is observed when axons pass on into the 
superficial layers where both en passant and terminaux are present (Fig. 1). Predominance of boutons en passant in claustral axons in layer 4 has also been described by (LeVay, 1986).

\section{Electron microscopy}

Investigation with the EM revealed that some of the axonal varicosities were indeed synaptic boutons. However, another portion of these varicosities turned out to be simply filled with tumid mitochondria. To examine further the relation between swellings and synapses, a region of claustral axon in layer 4 was reconstructed from 41 serial sections (Fig. 1C). The reconstruction shows that the swellings identified at LM cannot be directly correlated with the existence of a synaptic bouton (Fig. $1 D$; supplemental Fig. 2, available at www.jneurosci. org as supplemental material).

One occurrence of a labeled apical dendrite has been observed. Its shape was consistent with the form of apical dendrites of presumed claustrum-projecting pyramidal cells in area 17 seen in earlier studies (Katz, 1987). The corresponding soma was not found. No labeled boutons were sampled in the vicinity of the labeled dendrite.

In summary, layers $1,2 / 3,4$, and 6 have been examined in the EM. Data from layer $2 / 3$ was taken from both animals, the claustral synapses from the other layers were collected from one of the two cats. In both animals, the quality of the tissue ultrastructure allowed easy identification of pre- and postsynaptic structures. The detection of claustral axons in the EM was difficult because of the great sparseness of labeled structures. As expected all synapses formed by labeled boutons were asymmetric. Some of the spines targeted by claustral boutons formed a second symmetric synapse with a nonlabeled structure. As expected from LM observations, claustral boutons were small. Similar to thalamic boutons (Anderson et al., 2009), they were crowded with synaptic vesicles and carried mitochondria (Fig. 2; supplemental Figs. 3, 4, available at www.jneurosci.org as supplemental material).

To identify the targets of claustral boutons, 30 synapses were investigated in layer 1, 64 in layer 2/3, 39 from layer 4 and 29 from layer 6 . When the target could not explicitly be determined in a single section, serial photographs were taken from the corresponding location. Claustral boutons formed synapses preferentially with spines in all layers investigated (Fig. $3 A$ ). In layer 4, the percentage of synapses formed with dendritic shafts is slightly higher than in the other lamina. Except for layer 2/3, all of the targeted dendritic shafts had the morphological properties of smooth (putative GABAergic) neurons.

To visualize and quantify synapse size, we reconstructed the postsynaptic densities (PSDs) from serial sections of claustral boutons in layers 1 and $2 / 3$ (Fig. 3B). Approximately one third of the

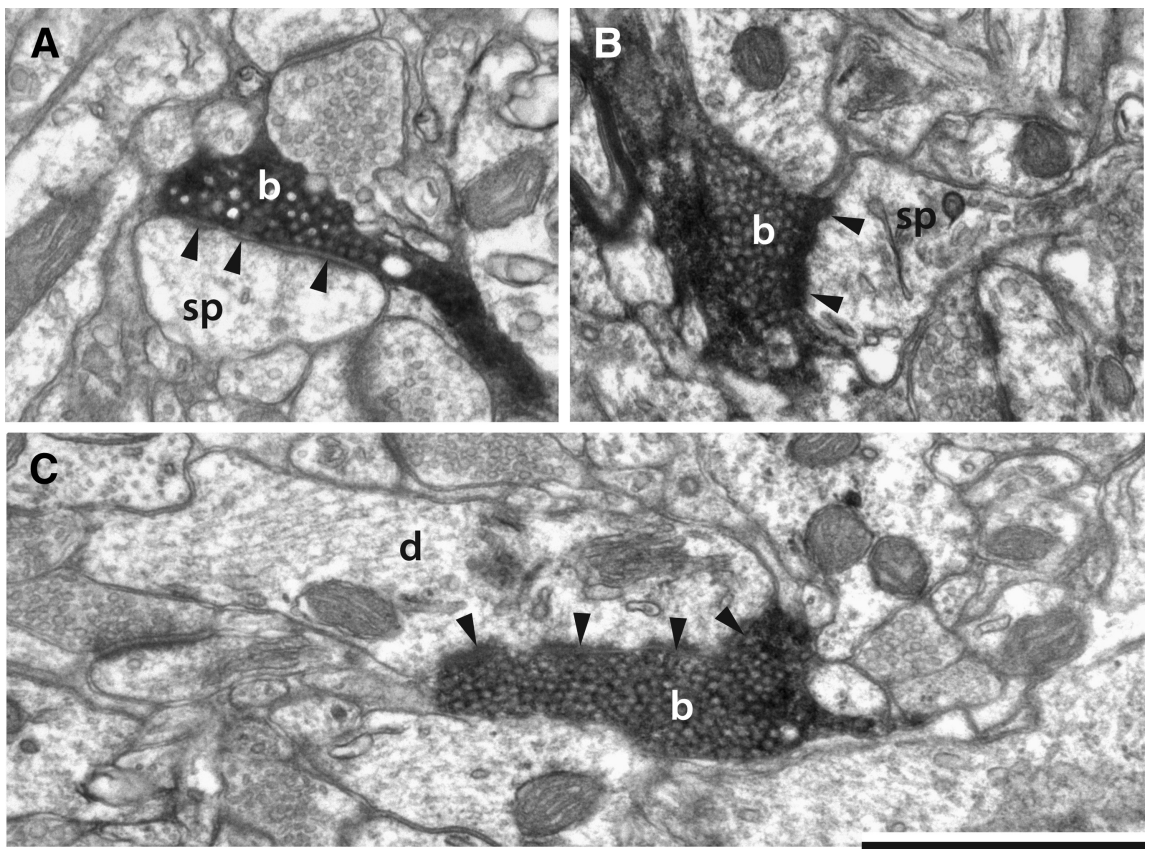

Figure 2. Electron micrographs of labeled claustral boutons. $A$, Claustral bouton (b) forming a perforated asymmetric synapse spine (sp) in layer 6 . The spine apparatus is clearly visible. C, Claustral bouton forming an asymmetric synapse (solid arrowheads)
A

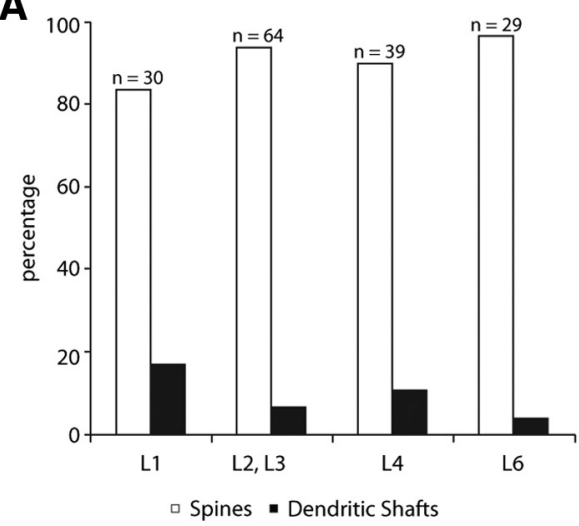

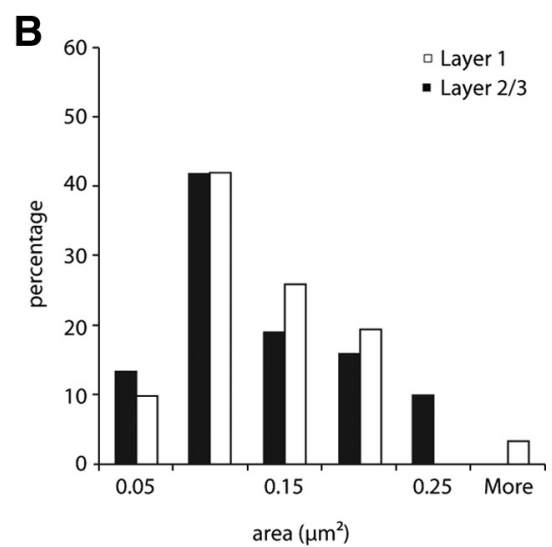

Figure 3. A, Histogram of synaptic targets of boutons in layer $1(n=30)$, layers 2 and $3(n=64)$, layer $4(n=39)$, and layer $6(n=29)$ of area V1 originating from neurons labeled in the ipsilateral claustrum. $\boldsymbol{B}$, Histogram of the distributions of postsynaptic areas $\left(\mu \mathrm{m}^{2}\right)$ formed by labeled claustral boutons in layer $1(n=31)$ and layers 2 and $3(n=31)$ of ipsilateral area 17.

synapses were perforated, the full extent of the PSDs are shown in the en face projections of Figure 4. Perforated postsynaptic densities were found in both spine and dendritic shaft synapses. In previous studies, perforated synapses have been found to be more common with spines (Anderson et al., 1998, 2009; Anderson and Martin, $2002,2005,2006)$. The synapse sizes of layers 1 and $2 / 3$ each had a mean of $0.1 \mu \mathrm{m}^{2}$, they were both normally distributed (Lillie test, $p_{\text {layer } 1}=0.4032, p_{\text {layer } 2 / 3}=0.0685$ ) and were not statistically different from each other (test, $p=0.8514$ ).

\section{Discussion}

In this study, we have examined the synapses formed by the claustrocortical projection, concentrating particularly on layers that were not examined by LeVay (1986) in his comparative study of the synaptic organization of claustral and thalamic afferents to area 17. Our LM observations agree with previous studies in the 
$\therefore$

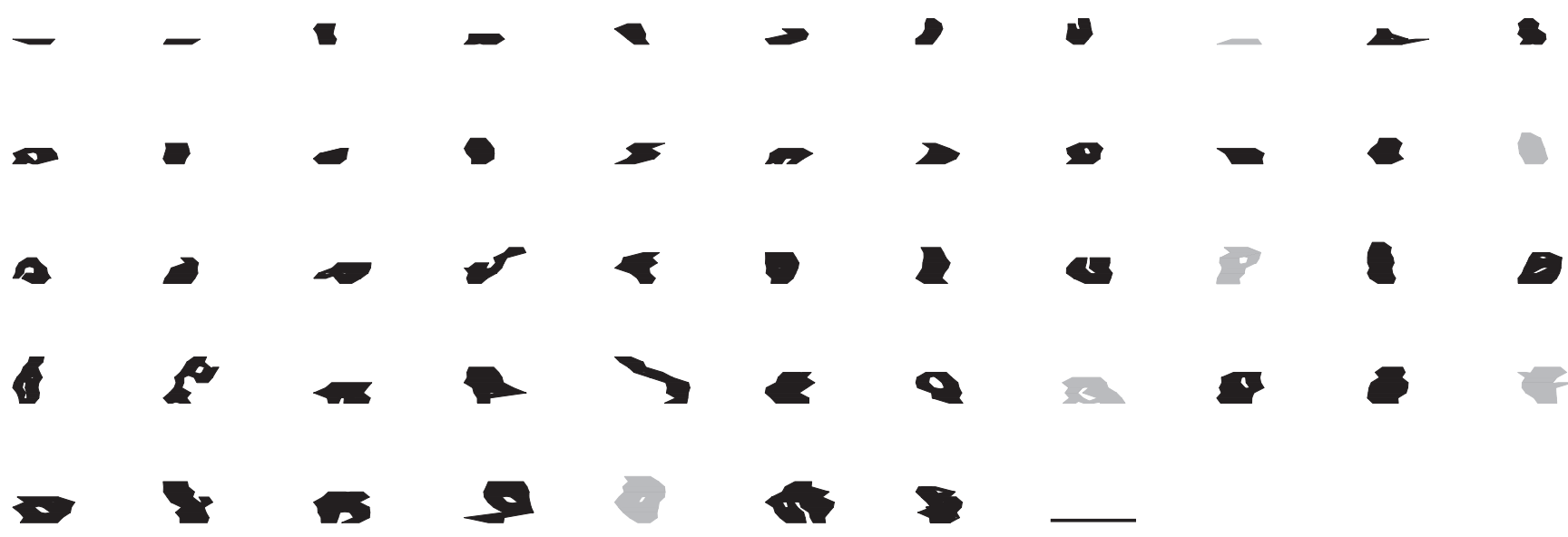

Figure 4. Two-dimensional projection of the reconstructed postsynaptic densities found on spines (black) and dendrites (gray) postsynaptic to claustral boutons found in layers 1, 2, and 3.Scale bar, $1 \mu \mathrm{m}$.

cat (Olson and Graybiel, 1980; LeVay and Sherk, 1981b; LeVay, 1986) and the tree shrew (Carey et al., 1980), which show that the claustrum projects to all layers of area 17. Unlike these earlier studies, we did not observe higher densities of label in layers 4 and 6. When LeVay (1986) made small ionophoretic injections with phaseolus lectin, similar in size to ours, he found a less obvious bias for layer 4 . He also raised the question of whether the axons innervating layer 4 were a different type to those innervating more superficial layers, because he found that the boutons in layer 1 were larger than in other layers and were distributed in horizontal strings. However, the axons he labeled were too incomplete for him to reach a satisfactory conclusion (LeVay, 1986).

Both Olson and Graybiel (1980) and LeVay (1986) remarked on the dense projection to layer 1 . This intrigued us, because we previously estimated that $90 \%$ of the asymmetric synapses in layer 1 do not originate from neurons in area 17 (Binzegger et al., 2004) and so we wondered whether the claustrum might not be a major contributor. The picture seen with the light microscope, however, is often somewhat deceptive, as evidenced by the projection of the thalamus to layer 4 , which is seen as dense in the light microscope, but which appears in the electron microscope as a sparse $5-6 \%$ of the excitatory synapses in layer 4 (Garey and Powell, 1971; LeVay, 1986; da Costa and Martin, 2009a). After comparing the results of large pressure injections of wheat germ agglutinin in the visual claustrum with similar injections in the lateral geniculate nucleus, LeVay (1986) concluded that the geniculocortical synapses greatly outnumbered the claustrocortical synapses in layer 4 . Unlike LeVay (1986), who found that layer 4 claustral boutons target dendritic shafts and dendritic spines equally, we found that the dominant targets are dendritic spines. The quantitative ultrastructural study of LeVay (1986) indicated that most of the labeled profiles in layers 1,4 , and 6 were not synaptic boutons, but axons. This branching as the axons enter the cortex obviously amplifies the label and gives a distorted impression of the relative density of innervation. We also found that the innervation of the superficial layers after our relatively punctate injections of the tracers was also very sparse, even in layer 1. Our data, along with those of LeVay (1986), provided us with no comfort that the claustrum was a major contributor of the missing asymmetric synapses in layer 1 .

Numerically, $\sim 240,000$ claustral projection neurons (LeVay and Sherk, 1981a,b) exist, which is similar in magnitude to the relay cells of the lateral geniculate nucleus $(340,000)$. Like the geniculate, the claustrum sprays its projections over most of the occipital cortex. Even if each claustrocortical neuron formed as many as 1500 synapses each in area 17, the claustral projection would still only account for $0.2 \%$ of the number of excitatory synapses in area 17 (Beaulieu and Colonnier, 1985; Binzegger et al., 2004). Given this tiny fraction, we needed to consider whether the size of the synapses was a critical factor in boosting the functional influence of the projection. Our results show that the claustral synapses in layer 2/3 (as in layer 1) are within the range of synapse sizes found in other cortical pathways. The conclusion is that the claustrocortical input to area 17 must be fractions of a percentage of the total complement of asymmetric synapses. This raises a significant question about how the claustrum exerts its influence on area 17.

In electrophysiological studies, Tsumoto and Suda (1982) stimulated the claustrum with short electrical pulses and found they could evoke spikes in one third of the 63 neurons they recorded in area 17 . They calculated that the conduction velocity of the axons evoking the shortest response was $\sim 2.4 \mathrm{~m} / \mathrm{s}$. This is $\sim 10$ times slower than the fastest thalamic axons and is consistent with the fine caliber of claustral axons $(<1 \mu$ m diameter). Electrical stimulation of cortical afferents always evokes an EPSPIPSP sequence, and the claustral afferents are no exception (Tsumoto and Suda, 1982). Tsumoto and Suda (1982) concluded that the claustrocortical path was exclusively excitatory and that the long latency inhibitory postsynaptic potentials they recorded were because of local circuit inhibitory neurons. Presumably, these inhibitory neurons were activated by Ptito and Lassonde (1981) who stimulated the claustrum with trains of electrical pulses and found that in $83 \%$ of the 63 cells they recorded, the magnitude of the response to visual stimulation and the spontaneous activity decreased. When Sherk and LeVay (1983) made kainic acid lesions in the claustrum, the only detectable effect was a weakening of the inhibitory end-zones of end-stopped receptive fields. This effect is unlikely to be mediated by monosynaptic connections since cortical targets of claustral axon do not show prominent end-inhibition (Boyapati and Henry, 1985). As LeVay (1986) pointed out, his finding with Sherk of reduced inhibition after claustral lesions was difficult to interpret given the ultrastructural evidence that the predominant target of the claustrocortical projection are the spines of excitatory cells. These observations imply that despite the ability of a single pulse of electrical stimulation to evoke spikes in many neurons, that the 
more asynchronous volley of spikes evoked during visual stimulation will exert a much more subtle effect.

Additional evidence for the subtle influence of the claustrocortical projections is that the receptive fields of claustral visual neurons summate up to lengths of 40 degrees, whereas most cortical neurons targeted by claustral afferents responded optimally to lengths of 3 degrees (Boyapati and Henry, 1985). Claustral cells are binocular and on average give similar responses to visual stimuli moving in both directions (Sherk and LeVay, 1981). Cells targeted by claustral afferents differ in some of these receptive field properties (Boyapati and Henry, 1985). For example, simple cells targeted by the claustrum are more acutely orientationtuned than claustral cells, as are complex cells. Layer 6 neurons targeted by the claustrum are more selective for direction preference than the general population (this effect is reversed in layer 4 neurons targeted by the claustrum). Among the possible targets of claustral axons are the layer 6 corticoclaustral neurons. However, the proportion of corticoclaustral cells in layer 6 is small $(<5 \%)$ (Katz, 1987), and their apical dendrite slim and unbranched above layer 4 . Statistically, this implies that even if the claustral afferents form synapses with corticoclaustral pyramidal cells, this will be a numerically tiny connection. The fact that the claustral neurons have large receptive fields and provide a sparse innervation of all layers in the different areas of occipital cortex points to an integrative role for the claustrum. This type of projection that targets all cortical layers is usually named a "lateral" connection between two equal areas in the hierarchy of interareal connectivity, instead of a feedback or feedforward projection. The claustrum is in a unique position as an intermediary in communication between cortical areas because it receives direct input from, and sends direct output to, the whole cortex. Acting as an intermediary, it can implement a "lateral" connection between any two or more cortical areas. In a belief propagation framework, the convergent input from cortex would provide the claustrum with an estimate of the current global percept or interpretation of the active cortical network. The claustral output to each cortical area then would act to enhance network activity that is consistent with the global perception or interpretation and would suppress activity that was inconsistent with the current interpretation. This functional view is consistent with the structural data we and others have provided and would in principle be testable in electrophysiological and functional imaging studies in awake primates.

\section{References}

Anderson JC, Martin KA (2002) Connection from cortical area V2 to MT in macaque monkey. J Comp Neurol 443:56-70.

Anderson JC, Martin KA (2005) Connection from cortical area V2 to V3 A in macaque monkey. J Comp Neurol 488:320-330.

Anderson JC, Martin KA (2006) Synaptic connection from cortical area V4 to V2 in macaque monkey. J Comp Neurol 495:709-721.

Anderson JC, Binzegger T, Martin KA, Rockland KS (1998) The connection from cortical area V1 to V5: a light and electron microscopic study. J Neurosci 18:10525-10540.

Anderson JC, da Costa NM, Martin KA (2009) The W cell pathway to cat primary visual cortex. J Comp Neurol 516:20-35.

Beaulieu C, Colonnier M (1985) A laminar analysis of the number of roundasymmetrical and flat-symmetrical synapses on spines, dendritic trunks, and cell bodies in area 17 of the cat. J Comp Neurol 231:180-189.

Binzegger T, Douglas RJ, Martin KA (2004) A quantitative map of the circuit of cat primary visual cortex. J Neurosci 24:8441-8453.

Boyapati J, Henry GH (1985) The character and influence of the claustral pathway to the striate cortex of the cat. Exp Brain Res 61:141-152.

Carey RG, Bear MF, Diamond IT (1980) The laminar organization of the reciprocal projections between the claustrum and striate cortex in the tree shrew, Tupaia glis. Brain Res 184:193-198.
Carman JB, Cowan WM, Powell TP (1964) The cortical projection upon the claustrum. J Neurol Neurosurg Psychiatry 27:46-51.

Clarke RH, Henderson EE (1914) Atlas of photographs of sections of the frozen cranium and brain of the cat. II. Frontal sections. J Psychol Neurol 21:273-277.

Crick FC, Koch C (2005) What is the function of the claustrum? Philos Trans R Soc Lond B Biol Sci 360:1271-1279.

da Costa NM, Martin KA (2009a) The proportion of synapses formed by the axons of the lateral geniculate nucleus in layer 4 of area 17 of the cat. J Comp Neurol 516:264-276.

da Costa NM, Martin KA (2009b) Selective targeting of the dendrites of corticothalamic cells by thalamic afferents in area 17 of the cat. J Neurosci 29:13919-13928.

Erickson SL, Lewis DA (2004) Cortical connections of the lateral mediodorsal thalamus in cynomolgus monkeys. J Comp Neurol 473:107-127.

Ettlinger G, Wilson WA (1990) Cross-modal performance: behavioural processes, phylogenetic considerations and neural mechanisms. Behav Brain Res 40:169-192.

Fernández-Miranda JC, Rhoton AL Jr, Kakizawa Y, Choi C, Alvarez-Linera J (2008) The claustrum and its projection system in the human brain: a microsurgical and tractographic anatomical study. J Neurosurg 108:764774.

Fiala JC (2005) Reconstruct: a free editor for serial section microscopy. J Microsc 218:52-61.

Garey LJ, Powell TP (1971) An experimental study of the termination of the lateral geniculo-cortical pathway in the cat and monkey. Proc R Soc Lond B Biol Sci 179:41-63.

Gray EG (1959) Axo-somatic and axo-dendritic synapses of the cerebral cortex: an electron microscope study. J Anat 93:420-433.

Katz LC (1987) Local circuitry of identified projection neurons in cat visual cortex brain slices. J Neurosci 7:1233-1249.

Kawano J (1998) Cortical projections of the parvocellular laminae C of the dorsal lateral geniculate nucleus in the cat: an anterograde wheat germ agglutinin conjugated to horseradish peroxidase study. J Comp Neurol 392:439-457.

Kemp JM, Powell TP (1970) The cortico-striate projection in the monkey. Brain 93:525-546.

LeVayS (1986) Synaptic organization of claustral and geniculate afferents to the visual cortex of the cat. J Neurosci 6:3564-3575.

LeVay S, Gilbert CD (1976) Laminar patterns of geniculocortical projection in the cat. Brain Res 113:1-19.

LeVay S, Sherk H (1981a) The visual claustrum of the cat. II. The visual field map. J Neurosci 1:981-992.

LeVay S, Sherk H (1981b) The visual claustrum of the cat. I. Structure and connections. J Neurosci 1:956-980.

Leventhal AG (1979) Evidence that the different classes of relay cells of the cat's lateral geniculate nucleus terminate in different layers of the striate cortex. Exp Brain Res 37:349-372.

Markowitsch HJ, Irle E, Bang-Olsen R, Flindt-Egebak P (1984) Claustral efferents to the cat's limbic cortex studied with retrograde and anterograde tracing techniques. Neuroscience 12:409-425.

Mathur BN, Caprioli RM, Deutch AY (2009) Proteomic analysis illuminates a novel structural definition of the claustrum and insula. Cereb Cortex 19:2372-2379.

Morys J, Narkiewicz O, Wisniewski HM (1993) Neuronal loss in the human claustrum following ulegyria. Brain Res 616:176-180.

Narkiewicz O (1964) Degenerations in the claustrum after regional neocortical ablations in the cat. J Comp Neurol 123:335-355.

Olson CR, Graybiel AM (1980) Sensory maps in the claustrum of the cat. Nature 288:479-481.

Ptito M, Lassonde MC (1981) Effects of claustral stimulation on the properties of visual cortex neurons in the cat. Exp Neurol 73:315-320.

Sherk H, LeVay S (1981) The visual claustrum of the cat. III. Receptive field properties. J Neurosci 1:993-1002.

Sherk H, LeVay S (1983) Contribution of the cortico-claustral loop to receptive field properties in area 17 of the cat. J Neurosci 3:2121-2127.

Tsumoto T, Suda K (1982) Effects of stimulation of the dorsocaudal claustrum on activities of striate cortex neurons in the cat. Brain Res 240:345-349.

Yoshida K, McCormack S, España RA, Crocker A, Scammell TE (2006) Afferents to the orexin neurons of the rat brain. J Comp Neurol 494:845-861. 